\title{
Le striga, mauvaise herbe parasite des céréales africaines : biologie et méthodes de lutte
}

\author{
A Olivier \\ Département de phytologie, faculté des sciences de l'agriculture et de l'alimentation, université Laval, \\ Sainte-Foy, Québec, G1K 7P4, Canada
}

(Reçu le 1er septembre 1995 ; accepté le 6 novembre 1995)

\begin{abstract}
Résumé - La mauvaise herbe parasite Striga hermonthica cause de graves dommages au sorgho, au mil et au maïs en Afrique. L'importance des pertes de rendement subies par les cultures est liée au mode de vie parasitaire du striga. Après avoir germé en réponse à des stimulants de germination libérés par la racine de son hôte, le striga développe en effet un genre de suçoir, l'haustorium, qui se fixe à une racine de la céréale, la pénètre et établit une connexion entre les vaisseaux conducteurs de sève des 2 plantes. Cela permet au parasite de prélever chez son hôte l'eau, les éléments minéraux et les substances organiques nécessaires à son développement. Le striga altère aussi l'activité photosynthétique de la céréale. Plusieurs méthodes de lutte, comme l'utilisation de stimulants de germination et d'herbicides, la pratique de rotations avec de faux hôtes et la sélection variétale n'ont donné jusqu'à maintenant que des résultats peu satisfaisants. Mais une meilleure compréhension des phénomènes de résistance au striga pourrait ouvrir de nouvelles voies dans la lutte contre le parasite. II devient par ailleurs urgent de multiplier les efforts pour vulgariser auprès des paysans les techniques actuellement disponibles pour réduire l'intensité des dommages causés par le striga.
\end{abstract}

\section{Striga hermonthica = striga / germination $/$ haustorium / méthode de lutte $/$ résistance}

Summary - Witchweed, a parasite of African cereal crops: biology and control methods. The parasite witchweed (Striga hermonthica) causes considerable yield losses in sorghum, pearl millet and maize in Africa. These losses are related to the parasitic nature of witchweed. The seed germinates in response to specific chemicals exuded by host plants. The germinated seed then develops a root structure, the haustorium, which attaches itself to the host root and penetrates its vascular tissue, establishing a connection that allows absorption of water, minerals and carbohydrates, which are essential for its development. The witchweed also affects the photosynthetic activity of the cereal crop. Control methods such as the use of germination stimulants and herbicides, rotation with 'false crops' and varietal selection gave disappointing results. A better understanding of resistance mechanisms is necessary to develop new control methods against the parasite. A better diffusion of available control methods is also urgently needed to help African farmers reduce crop damage caused by witchweed.

\section{Striga hermonthica $=$ witchweed $/$ germination $/$ haustorium $/$ control method $/$ resistance}




\section{INTRODUCTION}

La mauvaise herbe parasite Striga hermonthica (Del) Benth est devenue un véritable fléau dans plusieurs régions du continent africain. Elle s'attaque en effet à plusieurs cultures céréalières, auxquelles elle inflige des dommages qui provoquent une diminution importante de leur rendement (fig 1). Chez le sorgho, les pertes peuvent atteindre $70 \%$ (Doggett, 1988). Le striga a donc un impact socio-économique considérable. Les pertes de revenus de production sont estimées à plusieurs centaines de millions de dollars par année (Sauerborn, 1991). L'infestation atteint dans certaines localités un niveau si élevé que les paysans en sont réduits à abandonner leur culture principale. Cela peut avoir pour certains d'entre eux des conséquences dramatiques.

$S$ hermonthica n'est qu'une espèce parasite parmi un genre qui en comprend une quarantaine (Raynal-Roques, 1991). Mais c'est sans aucun doute celle qui cause le plus de dommages à ses hôtes. Elle s'attaque principalement au sorgho (Sorghum bicolor (L) Moench) et au mil (Pennisetum typhoides Burm), mais parasite aussi le maîs (Zea mays L), le fonio (Digitaria exilis Stapf), la canne à sucre (Saccharum officinarum $\mathrm{L}$ ) et le riz pluvial (Oryza sativa $\mathrm{L}$ ). Les autres espèces de striga qui entraînent des pertes appréciables chez les cultures sont
$S$ asiatica (L) Kuntze, qui parasite lui aussi plusieurs graminées, et $S$ gesnerioides (Willd) Vatke, qui est particulièrement virulent sur le niébé (Vigna unguiculata (L) Walp). Mais $S$ aspera (Willd) Benth serait également en voie de devenir un problème important dans les cultures de céréales en Afrique de l'Ouest (Sallé, 1991).

L'importance des dégâts causés par le striga vient de son mode de vie parasitaire. Très tôt dans sa vie, en effet, la mauvaise herbe développe une structure originale, l'haustorium, qui s'attache à une racine de la céréale, la pénètre et établit une connexion entre les vaisseaux conducteurs de sève des 2 plantes (fig 2). Cela permet au striga de se nourrir aux dépens de son hôte en prélevant chez ce dernier les éléments nutritifs nécessaires à sa croissance et à son développement. Un tel détournement peut affecter grandement le rendement de la céréale, tandis que le parasite prospère.

\section{UNE GERMINATION SOUMISE À DES EXIGENCES PARTICULIÈRES}

Vers la fin de la saison des pluies, chaque plant de $S$ hermonthica produit des milliers de graines. Ces graines traversent d'abord une période de dormance durant laquelle le taux potentiel de germination est très faible (Reid et Parker, 1979).

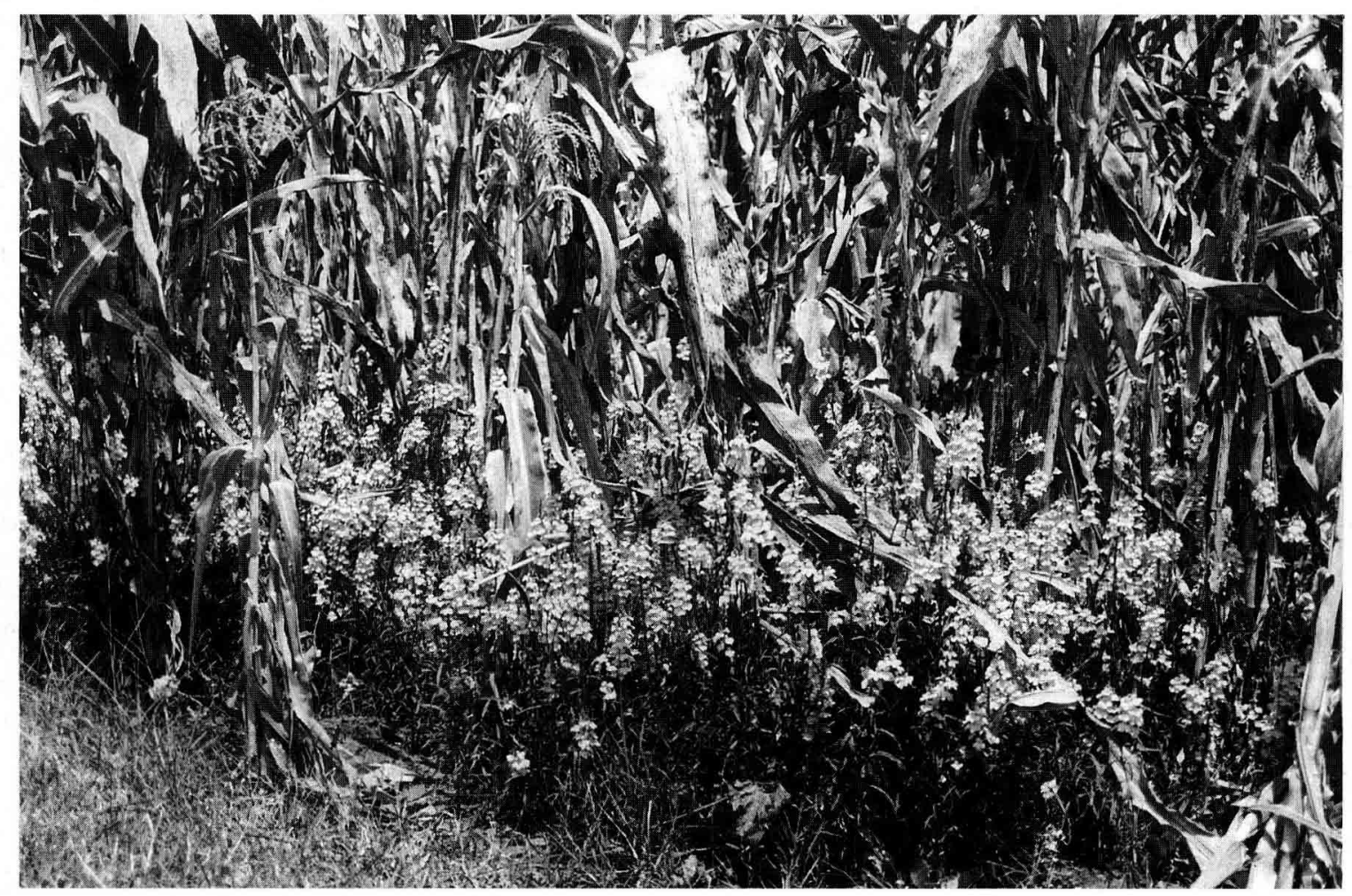

Fig 1. Un champ de maîs infesté par le striga au Kenya. 
Fig 2. Le cycle biologique du striga.

L'existence de cette période de dormance pourrait être le résultat d'une adaptation du striga au cycle vital de son hôte, puisqu'elle empêche la germination des graines entre le moment de la récolte de la céréale et celui de son semis à la saison suivante (Doggett, 1984). Ce n'est en effet qu'une fois la saison sèche terminée que les graines acquièrent la capacité de germer. Plusieurs jours de conditions humides sont nécessaires pour que le taux de germination soit maximal (Ransom et Njoroge, 1991). Cette période de conditionnement pourrait correspondre au temps nécessaire à l'imbibition des graines.

Mais le conditionnement de la graine ne suffit pas pour que la germination ait lieu. II faut aussi qu'une racine de la plante hôte se trouve à proximité de la graine de striga. Celle-ci ne germe en effet que lorsqu'elle est soumise à l'action d'un stimulant de germination libéré par cette racine. En l'absence de plante hôte, la germination demeure donc impossible. Mais la graine peut rester viable durant de nombreuses années, attendant les conditions propices pour germer (Vallance, 1950).

Plusieurs chercheurs ont essayé d'identifier les composés qui stimulent la germination de la graine de striga. Une première molécule a d'abord été isolée à partir de l'exsudat radiculaire d'une plante qui n'est pourtant pas l'hôte du striga, le coton (Gossypium hirsutum L) (Cook et al, 1966), puis caractérisée (Cook et al, 1972). On lui a donné le nom de strigol. Diverses équipes sont ensuite parvenues à synthétiser la molécule (Heather et al, 1974 ; MacAlpine et al, 1976) ou des analogues (Johnson et al, 1976). L'objectif était de pouvoir un jour produire ces composés en quantités suffisantes pour stimuler, en l'absence de plante hôte, la germination au champ. Comme le striga ne peut survivre sans se fixer à la racine d'une céréale, sa germination dans de telles conditions s'avérerait en effet suicidaire. Mais le coût prohibitif du strigol et de ses analogues empêche les paysans de les utiliser.

D'autres produits, comme l'éthylène, ont aussi montré des propriétés stimulantes sur la germination du striga. Mais ce n'est que plusieurs années après la découverte du strigol que des chercheurs ont réussi à isoler, chez une plante hôte, des composés actifs dans la stimulation de la germination (Chang et al, 1986 ; Netzly et al, 1988). On a donné à ces molécules hydrophobes, provenant de l'exsudat radiculaire du sorgho, et synthétisées pour la première fois par Sargent et Wangchareontrakul (1990), le nom de sorgoléones. L'exsudation continue de ces composés instables ne permettrait la germination des graines de striga qu'à une faible distance de la racine de la céréale, ce qui augmenterait les chances d'une fixation réussie de la plantule à son hôte (Fate et al, 1990). La croissance de la plantule de striga avant la fixation est en effet limitée à quelques millimètres en raison de ses réserves nutritives restreintes (Chang et Lynn, 1986).

II semble cependant exagéré d'attribuer aux seules sorgoléones le rôle de régulation de la germination des graines de striga. L'existence de stimulants hydrophiles très actifs est en effet 
depuis longtemps reconnue (Sunderland, 1960). La découverte récente de la présence de strigol dans l'exsudat radiculaire du sorgho et du maïs semble elle aussi remettre en question l'importance du rôle joué par les sorgoléones (Siame et al, 1993). II a par ailleurs été démontré que le strigol stimule la germination du striga en activant la synthèse d'éthylène (Babiker et al, 1993). Cela pourrait indiquer que l'existence d'une grande variété de substances pouvant stimuler la germination du striga viendrait de leur capacité commune à induire la production d'un composé actif unique (Press et al, 1990). Il est donc possible que les stimulants de germination n'aient pas une importance prépondérante dans la spécificité de la relation entre l'hôte et le parasite. Celle-ci pourrait plutôt dépendre de facteurs postérieurs à l'étape de germination.

\section{LA FORMATION D'UN LIEN ÉTROIT AVEC LA PLANTE HÔTE}

Au moment d'entrer en contact avec la racine de l'hôte, l'extrémité de la radicule du striga cesse brusquement son élongation et se transforme en un haustorium (Okonkwo, 1966). Plusieurs produits peuvent induire la différentiation de l'haustorium, y compris un composé, le 2,6-DMBQ, qui a été isolé à partir de la racine du sorgho (Chang, 1986). Mais cette étape d'induction ne serait pas déterminante dans la spécificité hôte-parasite, les composés actifs étant probablement communs à la majorité des plantes (Riopel et al, 1990).
La pénétration de la racine par le striga s'effectue sous l'initiative d'un groupe de cellules de I'haustorium (fig 3), et relèverait à la fois d'actions mécaniques (Dembélé, 1988) et enzymatiques (Olivier et al, 1991a). Une continuité vasculaire s'établit entre l'hôte et le parasite (Musselman et Dickison, 1975), ce qui permet d'expliquer le transfert d'eau et d'éléments minéraux de la céréale vers le striga (Rogers et Nelson, 1962). L'existence d'un transfert d'hydrates de carbone a également été mise en évidence (Ismail et Obeid, 1976).

Au tout début de sa croissance, le striga vit sous terre et dépend totalement de la culture pour son alimentation. Quelques semaines après la germination, la levée a lieu et le striga acquiert la capacité d'effectuer la photosynthèse. Mais son activité photosynthétique n'en demeure pas moins réduite (Shah et al, 1984), et le striga poursuit ses prélèvements à même les réserves de son hôte. Jusqu'à $85 \%$ du carbone présent chez le parasite à maturité peut être dérivé de la céréale (Graves et al, 1990). L'haustorium ne servirait toutefois pas seulement à collecter les substances provenant de l'hôte. II permettrait aussi de les entreposer et de les transformer avant de les transférer à la plantule en développement (Mallaburn et Stewart, 1987).

\section{UNE RELATION QUI ALTÈRE LA PHYSIOLOGIE DE LA CÉRÉALE}

Le potentiel hydrique du striga est plus négatif que celui de son hôte (Stewart et al, 1984), ce
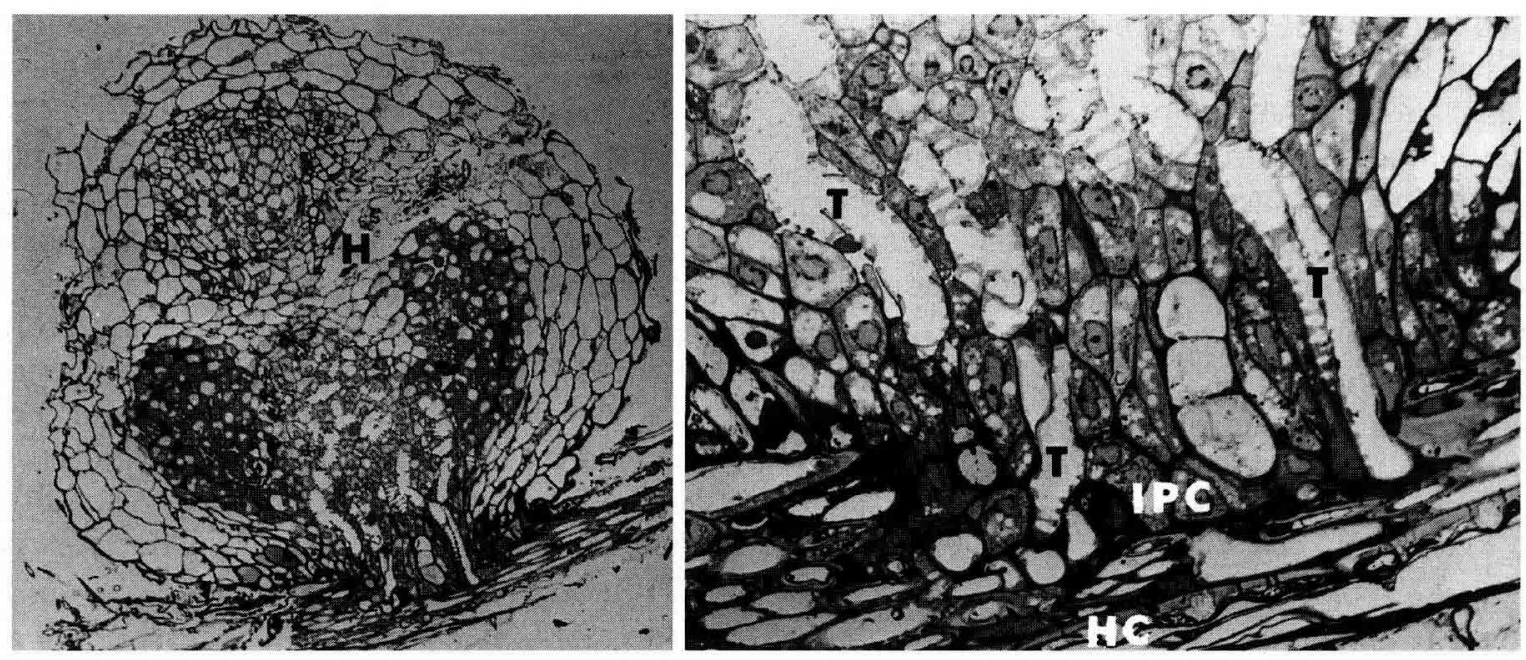

Fig 3. La connexion vasculaire entre le striga et le sorgho (coupe longitudinale). L'extrémité de la radicule du striga se transforme en un haustorium $(H)$ qui pénètre la racine de son hôte (photo de gauche) $(x 125)$. Un agrandissement (à droite) laisse voir les connexions établies entre les cellules du parasite (IPC) et celles de l'hôte (HC) (x 500) (Olivier et al, 1991a). 
qui pourrait faciliter le transfert d'eau vers le parasite. Son taux de transpiration est également très élevé ( $\mathrm{Ba}, 1983)$ et semble peu affecté par l'obscurité ou des stress hydriques modérés (Press et al, 1987c). Les stomates ne se ferment en effet que partiellement durant la nuit et restent ouverts lors d'un stress hydrique (Shah et al, 1987). Le striga maximise ainsi l'acquisition d'eau et d'éléments nutritifs. II paraît donc adapté au mode de vie parasitaire plutôt qu'aux conditions arides.

La culture en subit parfois les contrecoups. On a observé que l'efficacité d'utilisation de l'eau du sorgho était réduite par la présence du striga (Press et al, 1987b). Mais la compétition pour l'eau et les éléments minéraux ne serait importante que lorsque ces ressources deviennent particulièrement rares (Press et al, 1990). La compétition pour les substances organiques, pour sa part, semble avoir plus d'effet sur le rendement de la culture (Press et al, 1987a). Le prélèvement de carbone par le striga peut compter pour une part non négligeable des pertes de matière sèche chez l'hôte (Graves et al, 1990).

L'ampleur des dommages subis par la céréale dépendrait toutefois davantage d'une altération de son métabolisme que d'une compétition pour les ressources nutritives. L'activité photosynthétique du sorgho est fortement réduite à la suite d'une infection par le striga (Tuohy et al, 1987). Cette réduction pourrait être à l'origine de plus de $80 \%$ des pertes de rendement provoquées par le parasite (Graves et al, 1989).

Le striga provoque également chez son hôte des déséquilibres hormonaux. Une réduction de plus de $90 \%$ de la teneur en gibbérellines et en cytokinines, de même qu'une augmentation de la teneur en acide abscissique, a été notée chez le sorgho en réponse à l'infection (Drennan et El Hiweris, 1979). Ces modifications pourraient être responsables de la diminution de croissance des céréales observée sur le terrain.

\section{LA RECHERCHE DE MÉTHODES DE LUTTE EFFICACES}

Devant l'ampleur des dommages causés par le striga, de nombreux chercheurs ont tenté de développer diverses approches pour lutter contre le parasite. Dans les années 1970, on a cru que la solution viendrait du côté des composés chimiques. Aux États-Unis, l'incorporation d'éthylène dans les sols a permis de réduire le niveau des infestations en stimulant la germination suici- daire des graines de l'espèce $S$ asiatica (Eplee, 1975). Plusieurs herbicides se sont également avérés efficaces, parmi lesquels le 2,4-D, le paraquat, l'oxyfluorfen et les dinitroanilines (Langston et English, 1990 ; Eplee et al, 1991). Mais la phytotoxicité de l'herbicide envers la culture reste un problème difficile à maîtriser en raison de l'intimité de la relation entre le striga et la culture. L'efficacité des traitements est également restreinte par le fait que le parasite a déjà causé des dommages considérables à la culture avant d'émerger du sol. La création de variétés de céréales résistantes à un herbicide comme le glyphosate pourrait néanmoins permettre l'application d'herbicides sans risque d'affecter la culture (Gressel, 1992). À cause de son coût élevé, la lutte chimique demeure toutefois difficilement accessible pour la majeure partie des paysans africains.

Certaines techniques culturales peuvent également avoir des effets bénéfiques dans la lutte contre le striga. II existe une certaine controverse sur le rôle des engrais azotés, qui provient sans doute de la diversité des formes d'azote et des doses étudiées. II semble que des applications modérées d'engrais azotés aient peu d'effet sur le niveau d'infestation (Williams, 1961). Seules de fortes doses d'azote réduiraient les pertes de rendement causées par le striga en accroissant la vigueur de la céréale (Parker, 1984). Mais leur coût élevé rend leur emploi difficile à considérer en milieu paysan.

La réalisation de rotations avec certaines plantes a suscité un peu plus d'espoirs. En utilisant de faux hôtes comme le soja (Glycine max (L) Merr), le coton et le voandzou (Voandzeia subterranea $\mathrm{L}$ ), qui stimulent la germination $\mathrm{du}$ striga sans permettre sa fixation (Parkinson et al, 1987), on peut diminuer la quantité de graines de striga présentes dans le sol. La culture de plantes pièges, telle l'herbe du Soudan (Sorghum sudanense Stapf), qui permet l'attachement du parasite, mais qu'on détruit avant que celui-ci ait eu le temps de produire ses graines (Last, 1960), peut avoir un effet similaire. Mais il semble que de nombreuses années de rotation avec des plantes de ce type soient nécessaires pour que le degré d'infestation soit ramené à un niveau non dommageable. Or la disponibilité de terres arables est souvent insuffisante pour permettre aux paysans d'effectuer des rotations avec des plantes qui ne font pas partie de leur alimentation de base. Néanmoins, l'étude des faux hôtes pourrait amener une meilleure compréhension des mécanismes de résistance à la pénétration du striga. 
La pratique d'associations culturales a également été étudiée. L'association du sorgho avec le niébé pourrait limiter l'émergence du striga lorsque la couverture du niébé est importante (Carsky et al, 1994). Mais, de façon générale, les associations culturales ne semblent pas avoir un effet prononcé sur le niveau d'infestation.

C'est sans aucun doute l'utilisation de variétés de céréales résistantes au striga qui a créé le plus d'attentes. La résistance pourrait provenir d'une faible production de stimulants de germination ou de la présence de barrières à la pénétration de l'haustorium (Maiti et al, 1984). Plusieurs programmes de recherche ont été mis en place dans le but d'identifier des variétés résistantes, puis de transférer la résistance à des variétés à haut rendement (Ramaiah, 1987a). Certaines variétés de sorgho possédant un potentiel prometteur ont été identifiées (Olivier et al, 1991b ; Ramaiah, 1991). Mais les tentatives pour transférer la résistance se sont le plus souvent soldées par un échec (Ramaiah, 1987b). Si bien que les meilleures variétés actuellement disponibles pourraient bien être les variétés locales qui sont utilisées par les paysans depuis des générations. Sans être résistantes, plusieurs de ces variétés affichent une grande tolérance envers le parasite, qui leur permet de supporter sa présence sans que leur rendement soit affecté de façon trop importante.

Plus récemment, quelques chercheurs se sont intéressés aux méthodes de lutte biologique. Mais la plupart des travaux se sont jusqu'ici limi- tés à identifier les agents pathogènes et les insectes qui pourraient avoir un potentiel pour lutter contre le striga (Bashir, 1987). Des essais en serre ont toutefois révélé que le champignon pathogène Fusarium nygamai pourrait avoir un potentiel prometteur pour lutter contre le striga (Abbasher et Sauerborn, 1992). Un champignon mycorhizien, Glomus fasciculatum, aurait également la capacité de limiter l'infection par le striga (Gworgwor et Weber, 1992).

Pour le moment, il semble cependant que l'arrachage manuel reste bien souvent le seul recours du paysan. II s'agit toutefois d'une opération laborieuse dont les effets ne sont visibles qu'au bout de quelques années. L'arrachage manuel serait particulièrement efficace lorsqu'il est pratiqué avant la fioraison du striga, lors d'infestations légères, puisqu'il empêche la constitution d'une grande banque de graines dans le sol. Mais lors de graves infestations, l'arrachage interviendrait trop tard dans le cycle vital du parasite pour permettre une amélioration du rendement de la céréale (Ogborn, 1984). Les paysans qui arrachent le striga dès sa levée sont sans doute ceux qui ont le plus de chance de maintenir l'infestation à un niveau tolérable.

\section{CONCLUSION}

À première vue, le bilan de quelques décennies de recherche sur le striga peut paraître peu reluisant. II semble en effet n'y avoir encore que peu

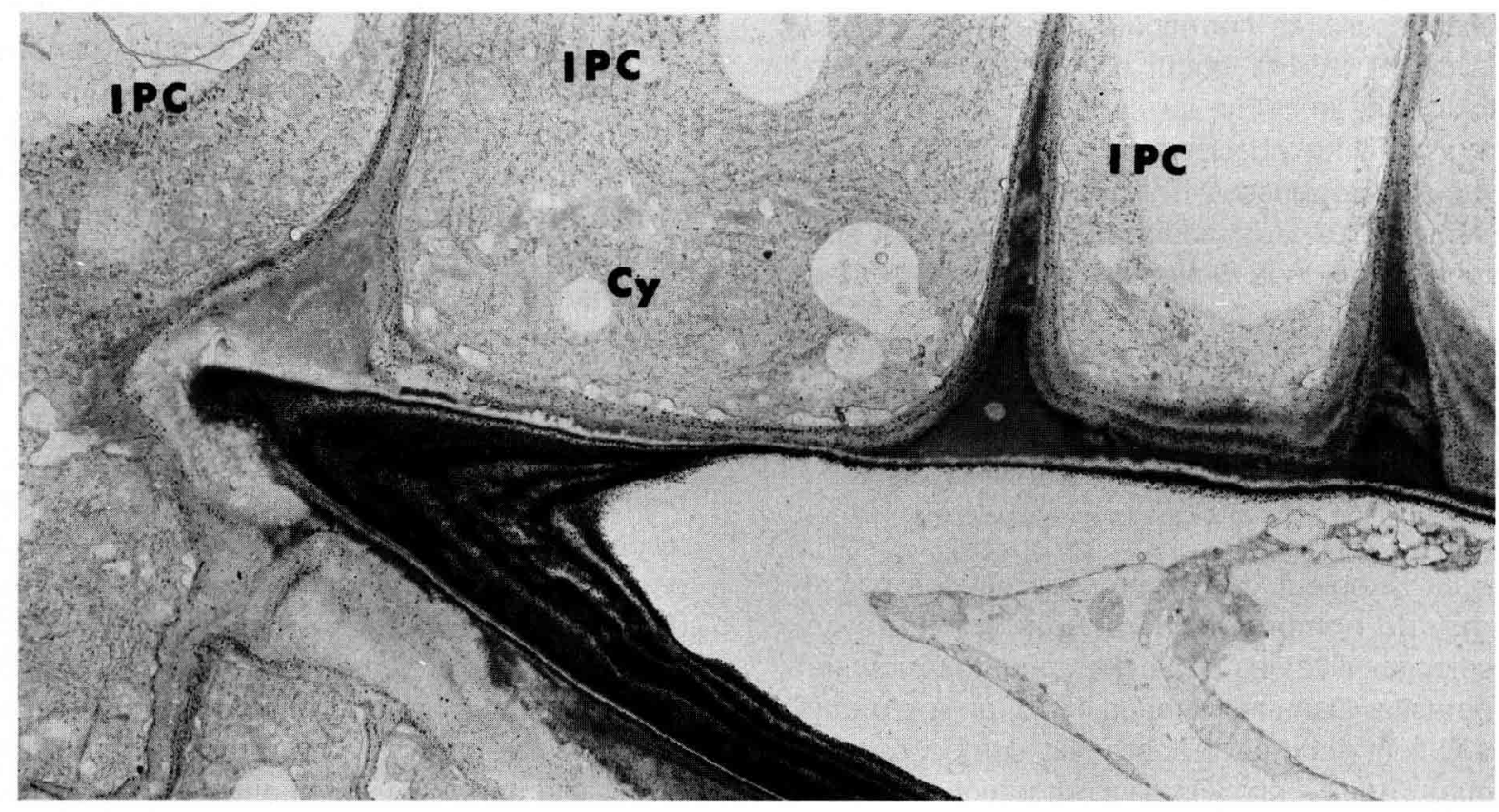

Fig 4. L'accumulation de couches successives de matériel cellulosique dans une cellule de sorgho en contact avec des cellules de striga (IPC) pénétrant la racine (x 5 400) (Olivier et al, 1991a). 
de moyens concrets de remédier aux problèmes engendrés par le parasite. Pourtant, si la méthode de lutte miracle reste encore à trouver, il ne faudrait pas dénigrer pour autant les progrès réalisés dans le développement de pratiques pouvant réduire les dégâts causés par le striga. L'utilisation de variétés tolérantes, la rotation avec de faux hôtes lorsque cela est envisageable et l'arrachage manuel ont chacun leur mérite. Sans doute le succès passe-t-il par une approche qui intégrerait chacune de ces pratiques.

Récemment, on a pu montrer que la résistance à la pénétration du striga chez le sorgho pouvait dépendre de l'accumulation dans certaines cellules de matériel néo-pariétal riche en cellulose (fig 4), de même que du recouvrement des parois par un matériel dense contenant vraisemblablement de grandes quantités de phénols (Olivier et al, 1991a). II se pourrait que la relation entre le striga et ses hôtes présente beaucoup de similitude avec les relations hôte-pathogène, et que la production de phénols, de phytoalexines, de protéines $\mathrm{Pr}$, de glycoprotéines riches en hydroxyproline et d'inhibiteurs d'enzymes puisse jouer un rôle aussi important dans la résistance d'une plante à l'infection par le striga que dans la résistance à un champignon pathogène. Cela ouvre la voie à un vaste champ d'études moléculaires qui pourraient déboucher sur l'identification et la caractérisation des gènes impliqués dans la résistance au striga.

D'ici là, il ne faudrait toutefois pas oublier que des milliers de paysans sont toujours aux prises avec la mauvaise herbe. II est donc nécessaire de leur donner dès maintenant les outils pour réduire les dommages causés à leurs cultures. La réalisation de cet objectif passe bien sûr par des investissements importants en recherche. Mais un effort accru pour que les connaissances acquises par les chercheurs soient vulgarisées auprès des agronomes, des techniciens agricoles, des formateurs ruraux et des paysans devient également plus que jamais nécessaire.

\section{RÉFÉRENCES}

Abbasher AA, Sauerborn J (1992) Fusarium nygamai, a potential herbicide for Striga hermonthica control in sorghum. Biol Control 2, 291-296

Ba AT (1983) Biologie du parasitisme chez 2 scrophulariacées tropicales: Striga hermonthica (Del) Benth et Striga gesnerioides (Willd) Vatke. Thèse de doctorat, université Cheikh Anta Diop, Dakar
Babiker AGT, Ejeta G, Butler LG, Woodson WR (1993) Ethylene biosynthesis and strigol-induced germination of Striga asiatica. Physiol Plantarum 88, 359365

Bashir MO (1987) The potential for biocontrol of witchweeds. In : Parasitic Weeds in Agriculture. Vol 1. Striga (LJ Musselman, ed), CRC Press, Inc, Boca Raton, 183-206

Carsky RJ, Singh L, Ndikawa R (1994) Suppression of Striga hermonthica on sorghum using a cowpea intercrop. Expl Agric 30, 349-358

Chang M (1986) Isolation and characterization of semiochemicals involved in host recognition in Striga asiatica. Ph D Thesis, University of Chicago, Chicago, IL

Chang M, Lynn DG (1986) The haustorium and the chemistry of host recognition in parasitic angiosperms. J Chem Ecol 12, 561-579

Chang M, Netzly DH, Butler LG, Lynn DG (1986) Chemical regulation of distance: characterization of the first natural host germination stimulant for Striga asiatica. J Am Chem Soc 108, 7858-7860

Cook CE, Whichard LP, Turner B, Wall ME, Egley GH (1966) Germination of witchweed (Striga asiatica Lour): isolation and properties of a potential stimulant. Science 154, 1189-1190

Cook CE, Whichard LP, Wall ME, Egley GH, Coggan P, Luhan PA, McPhail AT (1972) Germination stimulants. II. The structure of strigol - a potent seed germination stimulant for witchweed (Striga lutea Lour). J Am Chem Soc 94, 6198-6199

Dembélé B (1988) Aspects biologiques et agronomiques de 2 scrophulariacées parasites tropicales : Striga hermonthica (Del) Benth et Striga gesneroides (Willd) Vatke. Thèse de doctorat, ENSAM, Montpellier

Doggett H (1984) Striga - its biology and control. An overview. In : Proceedings of the Workshop on Biology and Control of Striga (ES Ayensu et al, eds), CRDI/ICSU Press, Dakar, 27-36

Doggett H (1988) Sorghum. Longman Scientific and Technical IDRC, New York

Drennan DSH, El Hiweris SO (1979) Changes in growth regulating substances in Sorghum vulgare infected by Striga hermonthica. In : Proceedings of the Second Symposium on Parasitic Weeds (LJ Musselman et al, eds), North Carolina State University, Raleigh, NC, 144-155

Eplee RE (1975) Ethylene: a witchweed seed germination stimulant. Weed Sci 23, 433-436

Eplee RE, Westbrooks RG, Norris RS (1991) Chemical control of Striga. In : Combating Striga in Africa. Proceedings of the International Workshop organized by IITA, ICRISAT and IDRC (SK Kim, ed), IITA, Ibadan, 61-68

Fate G, Chang M, Lynn DG (1990) Control of germination in Striga asiatica: chemistry of spatial definition. Plant Physiol 93, 201-207

Graves JD, Press MC, Stewart GR (1989) A carbon balance model of the sorghum-Striga hermonthica 
host-parasite association. Plant Cell Environ 12, 101-107

Graves JD, Wylde A, Press MC, Stewart GR (1990) Growth and carbon allocation in Pennisetum typhoides infected with the parasitic angiosperm Striga hermonthica. Plant Cell Environ 13, 367-373

Gressel J (1992) Addressing real weed science needs with inovation. Weed Technol 6, 509-525

Gworgwor NA, Weber HC (1992) Preliminary observations on control of Striga hermonthica (Del) Benth by vesicular-arbuscular mycorrhiza in sorghum (Sorghum bicolor (L) Moench). In : Compte rendu du IXe colloque international sur la biologie des mauvaises herbes, ANPP, Paris, 173-182

Heather JB, Mittal RSD, Sih CJ (1974) The total synthesis of d-strigol. J Am Chem Soc 96, 1976-1977

Ismail AMA, Obeid M (1976) A study of assimilation and translocation in Cuscuta hyalina Heyne ex Roth, Orobranche ramosa $L$ and Striga hermonthica Benth. Weed Res 16, 87-92

Johnson AW, Rosebery G, Parker C (1976) A novel approach to Striga and Orobanche control using synthetic germination stimulants. Weed Res 16 , 223-227

Langston TA, English TJ (1990) Vegetative control of witchweed and herbicide evaluation of techniques. In : Witchweed Research and Control in the United States (PF Sand et al, eds), WSSA, Champaign, IL, 107-113

Last FT (1960) Effect of cultural treatments on the incidence of Striga hermonthica (Del) Benth and yields of sorghum in the Sudan: field experiments $1957 / 8$. Ann Appl Biol 48, 207-229

MacAlpine GA, Raphael RA, Shaw A, Taylor AW, Wild $\mathrm{HJ}$ (1976) Synthesis of the germination stimulant ( \pm )-strigol. J Chem Soc, Perkin Trans I, 410-416

Maiti RK, Ramaiah KV, Bisen SS, Chidley VL (1984) A comparative stud of the haustorial development of Striga asiatica (L) Kuntze on sorghum cultivars. Ann Bot 54, 447-457

Mallaburn PS, Stewart GR (1987) Haustorial function in Striga: comparative anatomy of $S$ asiatica $(L)$ Kuntze and $S$ hermonthica (Del) Benth (Scrophulariaceae). In : Proceedings of the 4th International Symposium on Parasitic Flowering Plants (HC Weber, W Forstreuter, eds), PhilippsUniversity, Marburg, 523-536

Musselman LJ, Dickison WC (1975) The structure and development of the haustorium in parasitic Scrophulariaceae. Bot J Linn Soc 70, 183-212

Netzly DH, Riopel JL, Ejeta G, Butler LG (1988) Germination stimulants of witchweed (Striga asiatica) from hydrophobic root exudate of sorghum (Sorghum bicolor). Weed Sci 36, 441-446

Ogborn JEA (1984) Striga: research priorities with specific reference to agronomy. In : Proceedings of the Workshop on Biology and Control of Striga (ES Ayensu et al, eds), CRDI/ICSU Press, Dakar, 195212
Okonkwo SNC (1966) Studies on Striga senegalensis Benth. I. Mode of host-parasite union and haustorial structure. Phytomorphology 16, 453-463

Olivier A, Benhamou N, Leroux GD (1991a) Cell surface interactions between sorghum roots and the parasitic weed Striga hermonthica: cytochemical aspects of cellulose distribution in resistant and susceptible host tissues. Can J Bot 69, 1679-1690

Olivier A, Ramaiah KV, Leroux GD (1991b) Selection of sorghum (Sorghum bicolor (L) Moench) varieties resistant to the parasitic weed Striga hermonthica (Del) Benth. Weed Res 31, 219-225

Parker C (1984) The influence of Striga species on sorghum under varying nitrogen fertilization. In : Proceedings of the Third International Symposium on Parasitic Weeds (C Parker et al, eds), ICARDIAIPSPRG, Aleppo, 90-98

Parkinson V, Efron Y, Bello L, Dashiell K (1987) Trap crops as a cultural measure in Striga control in Africa. FAO Plant Protect Bull 35, 51-54

Press MC, Shah N, Tuohy JM, Stewart GR (1987a) Carbon isotope ratios demonstrate carbon flux from C4 host to C3 parasite. Plant Physiol 85, 1143-1145

Press MC, Tuohy JM, Stewart GR (1987b) Gas exchange characteristics of the sorghum-Striga host-parasite association. Plant Physiol 84, 814819

Press MC, Tuohy JM, Stewart GR (1987c) Leaf conductance, transpiration and relative water content of Striga hermonthica (Del) Benth and Striga asiatica (L) Kuntze (Scrophulariaceae) and their host Sorghum bicolor (L) Moench (Gramineae). In : Proceedings of the 4th International Symposium on Parasitic Flowering Plants (HC Weber, W Forstreuter, eds), PhilippsUniversity, Marburg, 631-636

Press MC, Graves JD, Stewart GR (1990) Physiology of the interaction of angiosperms parasites and their higher plant hosts. Plant Cell Environ 13, 91-104

Ramaiah KV (1987a) Breeding cereal grains for resistance to witchweed. In : Parasitic weeds in agriculture. Vol 1. Striga (LJ Musselman, ed), CRC Press Inc, Boca Raton, 227-242

Ramaiah KV (1987b) Control of Striga and Orobanche species - a review. In : Proceedings of the 4th International Symposium on Parasitic Flowering Plants (HC Weber, W Forstreuter, eds), PhilippsUniversity, Marburg, 637-664

Ramaiah KV (1991) Breeding for Striga resistance in sorghum and millet. In : Combating Striga in Africa. Proceedings of the International Workshop organized by IITA, ICRISAT and IDRC (SK Kim, ed), IITA, Ibadan, 75-80

Ransom JK, Njoroge J (1991) Seasonal variation in ethylene-induced germination of Striga hermonthica in Western Kenya. In : Proceedings of the Fifth International Symposium of Parasitic Weeds (JK Ransom et al, eds), CIMMYT, Nairobi, 391-396

Raynal-Roques A (1991) Diversification in the genus Striga. In : Proceedings of the Fifth International 
Symposium of Parasitic Weeds (JK Ransom et al, eds), CIMMYT, Nairobi, 251-261

Reid DC, Parker C (1979) Germination requirements of Striga species. In : Proceedings of the Second Symposium on Parasitic Weeds (LJ Musselman et al, eds), North Carolina State University, Raleigh, NC, 202-210

Riopel JL, Baird WV, Chang M, Lynn DG (1990) Haustorial development in Striga asiatica. In : Witchweed Research and Control in the United States (PF Sand et al, eds), WSSA, Champaign, IL, 27-36

Rogers WE, Nelson RR (1962) Penetration and nutrition of Striga asiatica. Phytopathology 52, 10641070

Sallé G (1991) Striga research for West Africa sponsored by the European Economic Community. In : Combating Striga in Africa. Proceedings of the International Workshop organized by IITA, ICRISAT and IDRC (SK Kim, ed), IITA, Ibadan, 117-121

Sargent MV, Wangchareontrakul S (1990) The synthesis of the first natural host stimulant for Striga asiatica (witchweed). J Chem Soc, Perkin Trans I, 14291434

Sauerborn J (1991) The economic importance of the phytoparasites Orobanche and Striga. In : Proceedings of the Fifth International Symposium of Parasitic Weeds (JK Ransom et al, eds), CIMMYT, Nairobi, 137-143

Shah N, Tuohy J, King G, Stewart GR (1984) A biochemical characterisation of Striga hermonthica. $I n$ : Proceedings of the Third International Symposium on Parasitic Weeds (C Parker et al, eds), ICARDAIPSPRG, Aleppo, 74-80

Shah N, Smirnoff N, Stewart GR (1987) Photosynthesis and stomatal characteristics of Striga hermonthica in relation to its parasitic habit. Physiol Plantarum 69, 699-703

Siame BA, Weerasuriya Y, Wood K, Ejeta G, Butler LG (1993) Isolation of strigol, a germination stimulant for Striga asiatica, from host plants. J Agric Food Chem 41, 1486-1491

Stewart GR, Nour J, Macqueen M, Shah N (1984) Aspects of the biochemistry of Striga. In : Proceedings of the Workshop on Biology and Control of Striga (ES Ayensu et al, eds), CRDI/ICSU Press, Dakar, 161-178

Sunderland N (1960) The production of the Striga and Orobanche germination stimulants by maize roots. I. The number and variety of stimulants. $J$ Exp/ Bot $11,236-245$

Tuohy JM, Press MC, Stewart GR (1987) $\mathrm{CO}_{2}$ fixation and water relations of Striga-infected sorghum. In : Proceedings of the 4th International Symposium on Parasitic Flowering Plants (HC Weber, W Forstreuter, eds), Philipps-University, Marburg, 775780

Vallance KB (1950) Studies on the germination of seeds of Striga hermonthica. I. The influence of moisture treatment, stimulant dilution and after-ripening on germination. Ann Bot 14, 347-363

Williams CN (1961) Effect of inoculum size and nutrition on the host/parasite relations of Striga senegalensis on sorghum. Plant Soil 15, 1-12 